\title{
Theory of the reactant-stationary kinetics for a coupled auxiliary enzyme assay
}

\author{
Justin Eilertsen $^{\mathrm{a}}$, Wylie Stroberg ${ }^{\mathrm{a}}$, Santiago Schnell ${ }^{\mathrm{a}, \mathrm{b}, \mathrm{c}, 1}$ \\ ${ }^{a}$ Department of Molecular $\&$ Integrative Physiology, University of Michigan Medical School, \\ Ann Arbor, MI 48109, USA \\ ${ }^{b}$ Department of Computational Medicine $\&$ Bioinformatics, University of Michigan Medical \\ School, Ann Arbor, MI 48109, USA \\ ${ }^{c}$ Brehm Center for Diabetes Research, University of Michigan Medical School, Ann Arbor, \\ $M I$ 48105, USA
}

\begin{abstract}
A theoretical analysis is performed on the nonlinear ordinary differential equations that govern the dynamics of a coupled auxiliary enzyme catalyzed reaction. The assay consists of a non-observable reaction and an indicator (observable) reaction, where the product of the first reaction is the enzyme for the second. Both reactions are governed by the single substrate, single enzyme MichaelisMenten reaction mechanism. Using singular perturbation methods, we derive asymptotic solutions that are valid under the quasi-steady-state and reactantstationary assumptions. In particular, we obtain closed form solutions, analogous to the Schnell-Mendoza equation for Michaelis-Menten type reactions, that approximate the evolution of the observable reaction. Conditions for the validity of the asymptotic solutions are also rigorously derived showing that these asymptotic expressions are applicable under the reactant-stationary kinetics.
\end{abstract}

Keywords: Coupled enzyme assay, time course experiments, timescale separation analysis, singular perturbation analysis, Schnell-Mendoza equation

Email address: schnells@umich.edu (Santiago Schnell)

${ }^{1}$ Corresponding author 


\section{Introduction}

Difficult detectable or non-observable enzyme reactions are frequently coupled with easily observable reactions to be studied in enzyme kinetic experiments; the hope is that the enzyme activity of the non-observable reaction can be measured by analyzing the progress curves of the secondary observable reaction. Traditionally, coupled assays are designed in the sequential form (see [1] for specific applications), in which the product of the non-observable reaction is a substrate that is catalyzed by a second, sequential enzyme. In addition to the well-studied sequential assay [2, 3, 4, 5, there is also the auxiliary enzyme assay. In this assay, the primary enzyme, $E_{1}$, reacts with the substrate $S_{1}$ to form an intermediate complex $C_{1}$ following the Michaelis-Menten (MM) [6] mechanism. The product of the primary reaction is thus the activated form of the secondary enzyme, $E_{2}$ :

$$
E_{1}+S_{1} \stackrel{k_{1}}{\rightleftharpoons} C_{1} \stackrel{k_{2}}{\rightarrow} E_{1}+E_{2} .
$$

The first (primary) reaction (1) represents a non-observable enzyme catalyzed reaction. In the secondary reaction, the substrate $S_{2}$ binds with the enzyme $E_{2}$ to form a complex $C_{2}$, which will synthesize the product, $P$, and release the enzyme, $E_{2}$ in the catalytic step of the reaction:

$$
E_{2}+S_{2} \stackrel{k_{3}}{\rightleftharpoons} C_{2} \stackrel{k_{4}}{\longrightarrow} E_{2}+P .
$$

Mechanism (2) represents the observable reaction, which is is generally known as the indicator reaction. In this coupled enzyme assay, the product enzyme, $E_{2}$, of the non-observable reaction is known as an auxiliary enzyme, because it 5 provides support to measure the non-observable reaction through the indicator reaction. In the above chemical steps, $k_{1}, k_{-1}, k_{3}, k_{-3}$ are microscopic rate constants, and $k_{2}, k_{4}$ are catalytic constants. 
The auxiliary reaction mechanism (1)-(2) occurs naturally in coagulation cascades [7. As a distinct example, the activation of protein $\mathrm{C}(P C)$ by thrombin $(T)$ follows a reaction consistent with (1):

$$
T+P C \stackrel{k_{1}}{\rightleftharpoons}{ }^{k_{2}}
$$

where " $A P C$ " denotes the activated form of $P C$. In the experimental assay, the activated enzyme APC then catalyzes a substrate (S). Assuming $S$ is specific to $A P C$ and thus does not bind with $T$, the secondary, observable reaction follows the form of 2 ,

$$
A P C+S \stackrel{k_{3}}{\rightleftharpoons} S A P C \stackrel{k_{4}}{\longrightarrow} A P C+P .
$$

Experimentally, the kinetics of the non-observable reaction is measured by decoupling the analysis of progress curves by adding excessive concentrations of the primary enzyme, and making the first reaction pseudo-first order [7]. There has been a reasonable amount of literature that features kinetic modeling of coagulation cascades $8,9,10$, but complex cascade kinetic models have limited applicability to the parameter estimation of enzyme assays, like the auxiliary reaction mechanism (1)-22. Derivation of rate equations for coupled enzyme assays has been primarily limited to the sequential enzyme system [4, 5, 2], rather than the auxiliary enzyme assay due to the complex non-linear coupling of the differential equations governing the kinetic of the reaction mechanism.

Under appropriate conditions, the rate of substrate depletion for the nonobservable reaction is described by the MM equation,

$$
\dot{s}_{1}=-\frac{V_{1}}{K_{M_{1}}+s_{1}} s_{1}
$$

20

where $s_{1}$ is the concentration of $S_{1}, K_{M_{1}}=\left(k_{-1}+k_{2}\right) / k_{1}$ is the Michaelis constant, and $V_{1}=k_{2} e_{1}^{0}$ is limiting rate of the reaction, which is dependent on 
both the catalytic constant $k_{2}$ and the initial concentration of $E_{1}$ (the initial concentration of $E_{1}$ is denoted as $\left.e_{1}^{0}\right)$. Of great interest to both theoreticians [11, 12 and experimentalists is the estimation of the constants $K_{M_{1}}$ and $V_{1}$ 25 from the so-called inverse problem. The inverse problem is carried out in two stages: First, experimental data is produced in the form of a progress curve for either $s_{1}$ or $p$ (we have used lower case letters to denote the concentrations of $S_{1}$ and $P$ respectively). Second, the experimental data is then used to try and estimate both $K_{M_{1}}$ and $V_{1}$ by optimally fitting the model (5) through the utilization of either a deterministic (i.e., such as Levenburg-Marquardt) or a stochastic (Markov Chain Monte Carlo) algorithm. In general, one seeks to estimate kinetic constants with an expression contains the fewest number of parameters; this is why the MM equation is more attractive than the complete set of mass action equations. The MM equation is what is known as a reduced model, and it is reduced in the sense that it contains fewer variables $\left(s_{1}\right.$ versus $s_{1}$ and $\left.c_{1}\right)$ and fewer parameters $\left(K_{M_{1}}\right.$ and $V_{1}$ versus $k_{1}, k_{-1}$ and $\left.k_{2}\right)$.

The inverse problem presents a unique challenge for both experimentalists and theorists. First, the parameters that govern the enzyme activity of the non-observable reaction must somehow be determined from the indicator re40 action, since in a typical in vitro laboratory experiment, progress curves can only be generated for the indicator reaction. Second, a reduced model for coupled auxiliary enzyme reactions must be developed. The reduced model should, (1) decrease the number of variables, and (2) lessen the number of parameters needed to describe the time course of the complete auxiliary reaction.

1.1. Enzyme kinetic rate equations are reduced models resulting from scaling and simplification analysis

The MM equation is the result of a model reduction method known as slow manifold projection. The validity of the MM equation resides under the assumption that the single-enzyme, single-substrate reaction 1 has two intrinsic timescales. The first timescale is very short, and accounts for the rapid accumulation of the complex $C_{1}$. The second timescale is very long, and gives a rough 
measure of the time it takes for the completion of the reaction. Respectively, these timescales are known as fast and slow timescales. If these timescales are inherently present within (1), then the dimensionless mass action equations that model 1 can be written in the form

$$
\begin{aligned}
\dot{s}_{1} & =f_{1}\left(s_{1}, c_{1}\right) \\
\varepsilon \dot{c}_{1} & =f_{2}\left(s_{1}, c_{1}\right)
\end{aligned}
$$

where $\varepsilon$ is very small (i.e., $\varepsilon \ll 1$ ), and is proportional to the ratio of the fast timescale to the slow timescale. Differential equations in the form of (6) are called singularly perturbed differential equations, and they are ubiquitous in mathematical chemistry [13, 14, 15] and biology [16]. Thus, central to deriving a reduced model for the auxiliary reaction (using slow manifold projection) is the estimation of the slow and fast timescales for the non-observable reaction. This is challenging for coupled reactions, since the time to completion of the indicator reaction can occur before, after, or at approximately the same time as the non-observable reaction. Furthermore, it is unlikely that the relative speeds and completion time of the non-observable reaction (1) will be known. Thus, there is a need derive a reduced model that is general enough so that its validity is certain regardless of which reaction is fastest. Finally, the most desirable reduced model will be one in which a closed form solution is obtainable so that the reduced model may be expressed as an explicit function of time. This will eliminate the need to generate explicit progress curves for $s_{1}$, since the time course of $s_{1}$ is non-observable and consequently unknown.

\subsection{Goals of this work}

Up to date, the reduction theoretical analysis of auxiliary enzyme catalyzed reactions has been limited to first-order kinetics models [4, 5, 3, which has a limited validity in time course experiments [17]. Through singular perturbation methods, we will show that the complete (coupled) system that includes both the non-observable and indicator reaction can be reduced to a system of the 
form

$$
\begin{aligned}
& \dot{s}_{1}=f\left(s_{1} ; K_{M_{1}}, V_{1}\right) \\
& \dot{s}_{2}=g\left(s_{1}, s_{2} ; K_{M_{1}}, K_{M_{2}}, V_{1}, V_{2}\right)
\end{aligned}
$$

where $K_{M_{2}}=\left(k_{-3}+k_{4}\right) / k_{3}$ is the Michaelis constant of the indicator reaction, and $V_{2}=k_{4} s_{1}^{0}$ is the limiting rate of the indicator reaction $\left(s_{1}^{0}\right.$ denotes the initial concentration of $S_{1}$ ). The reduced model (7) admits closed-form solutions in the form of a Schnell-Mendoza equation [18; conditions for the validity of the reduced model will be established, and timescale estimates will be derived. In addition, we will exploit the geometry of the mathematical structure [19, 20] in extreme situations when the speeds of the reactions are significantly disparate; this will allow us to "simplify" the reduced model and obtain asymptotic solutions that are in some ways easier in form than both the general reduced model and the system of mass action equations. Finally, in Section 6 , we conclude with a brief discussion of the results and their relevance in possible future work involving the inverse problem.

\section{Derivation of the governing equations for the coupled auxiliary enzyme reactions}

Applying the law of mass action to coupled auxiliary enzyme reaction mechanism (1)-22 yields seven rate equations

$$
\begin{aligned}
& \dot{e_{1}}=-k_{1} e_{1} s_{1}+\left(k_{-1}+k_{2}\right) c_{1} \\
& \dot{s_{1}}=-k_{1} e_{1} s_{1}+k_{-1} c_{1} \\
& \dot{c_{1}}=k_{1} e_{1} s_{1}-\left(k_{-1}+k_{2}\right) c_{1} \\
& \dot{e_{2}}=k_{2} c_{1}-k_{3} e_{2} s_{2}+\left(k_{-3}+k_{4}\right) c_{2} \\
& \dot{s_{2}}=-k_{3} e_{2} s_{2}+k_{-3} c_{2} \\
& \dot{c_{2}}=k_{3} e_{2} s_{2}-\left(k_{-3}+k_{4}\right) c_{2} \\
& \dot{p}=k_{4} c_{2},
\end{aligned}
$$

where lowercase letters represent concentrations of the corresponding uppercase species. Typically, laboratory enzyme assays present the following initial 
conditions

$$
\left.\left(e_{1}, s_{1}, c_{1}, e_{2}, s_{2}, c_{2}, p\right)\right|_{t=0}=\left(e_{1}^{0}, s_{1}^{0}, 0,0, s_{2}^{0}, 0,0\right)
$$

By examining the system of rate equations (8), the coupled auxiliary enzyme reaction mechanism obeys three conservation laws:

$$
\begin{aligned}
& e_{1}(t)+c_{1}(t)=e_{1}^{0}, \\
& s_{1}(t)+c_{1}(t)+c_{2}(t)+e_{2}(t)=s_{1}^{0}, \\
& s_{2}(t)+c_{2}(t)+p(t)=s_{2}^{0} .
\end{aligned}
$$

Mathematically speaking, the solution trajectory to must lie on the intersection of the hyperplanes defined in $(10)$, which means the original sevendimensional problem can be reduced to a four-dimensional problem. Using (10a) and $10 \mathrm{~b}$ to decouple the enzyme concentrations, the redundancies in the system (8) are eliminated to yield

$$
\begin{aligned}
& \dot{s_{1}}=-k_{1}\left(e_{1}^{0}-c_{1}\right) s_{1}+k_{-1} c_{1} \\
& \dot{c_{1}}=k_{1}\left(e_{1}^{0}-c_{1}\right) s_{1}-\left(k_{-1}+k_{2}\right) c_{1} \\
& \dot{s_{2}}=-k_{3}\left(s_{1}^{0}-s_{1}-c_{1}-c_{2}\right) s_{2}+k_{-3} c_{2} \\
& \dot{c_{2}}=k_{3}\left(s_{1}^{0}-s_{1}-c_{1}-c_{2}\right) s_{2}-\left(k_{-3}+k_{4}\right) c_{2},
\end{aligned}
$$

where $e_{1}(t), e_{2}(t)$ and $p(t)$ are readily calculated once $s_{1}(t), c_{1}(t), s_{2}(t)$ and $c_{2}(t)$ are known.

\section{Rate expressions for the non-observable enzyme catalyzed reaction}

The rate equations $11 \mathrm{a}-1 \mathrm{~b}$ ) are uncoupled from $11 \mathrm{c}-(11 \mathrm{~d})$. These rate equations have the same structure to those of the single-substrate, single-enzyme reaction following the MM mechanism. Therefore, it is possible to derive rate equations to model the coupled auxiliary enzyme catalyzed reaction, and estimate its kinetic parameters using the general theory of the reactant-stationary assumption (RSA, [21]). The rate equations for the non-observable reaction are identical to those of the single substrate, single enzyme reaction following the MM mechanisms. 


\subsection{Review of the single substrate, single enzyme MM reaction}

Revisiting the analysis for the single-substrate, single-enzyme reaction, it has long been established that there there can be a rapid buildup of $c_{1}$ during an initial fast transient of the non-observable reaction. After this rapid buildup (where the rate of depletion of $c_{1}$ approximately equals its rate of formation) $c_{1}$ is assumed to be in a quasi-steady-state (QSS),

$$
\dot{c_{1}} \approx 0 \text { for } t>t_{c_{1}}
$$

The timescale $t_{c_{1}}$ is the time associated with the initial transient buildup of $c_{1}$,

$$
t_{c_{1}}=\frac{1}{k_{1}\left(K_{M_{1}}+s_{1}^{0}\right)} .
$$

The quasi-steady-state assumption (QSSA, 12), in combination with 11a)(11b), leads to the derivation of the well-known rate expressions,

$$
\begin{aligned}
& c_{1}=\frac{e_{1}^{0} s_{1}}{K_{M_{1}}+s_{1}} \\
& \dot{s_{1}}=-\frac{V_{1} s_{1}}{K_{M_{1}}+s_{1}},
\end{aligned}
$$

from which we see that the mass action equations $111 \mathrm{a}-11 \mathrm{~b})$ are reduced to a

differential-algebraic equation systems with one single differential equation for $s_{1}$.

Since equations 14a and (14b are only valid after the initial transient, $t_{c_{1}}$, it is necessary to define a boundary condition for $s_{1}$ at $t=t_{c_{1}}$. This is equivalent to the initial experimental condition for the initial rate or time course experiments. To find this condition, it can be assumed that there is a negligible decrease in $s_{1}$ during the initial transient. This is known as the RSA, and is expressed as

$$
s_{1}\left(t<t_{c_{1}}\right) \approx s_{1}^{0}
$$

The RSA provides an initial condition for 11a under the variable transformation $\hat{t} \mapsto t-t_{c_{1}}$. The mathematical expression $14 \mathrm{~b}$ is the MM equation, and 125 the system 14a $14 \mathrm{~b}$ governs the dynamics of the substrate $s_{1}$ and complex $c_{1}$ 
of the observable reaction under the QSS and RSA. The explicit closed-form solution of $(14 \mathrm{~b})$, with the initial condition $(15)$, is known as the Schnell-Mendoza equation [18, and is written in terms of the Lambert- $W$ function

$$
\frac{s_{1}(\hat{t})}{K_{M_{1}}}=W\left[\sigma_{1} \exp \left(\sigma_{1}-\eta_{1} \hat{t}\right)\right], \quad \sigma_{1}=\frac{s_{1}^{0}}{K_{M_{1}}}, \quad \eta_{1}=\frac{V_{1}}{K_{M_{1}}} .
$$

From the perspective of asymptotic theory, Schnell and Mendoza [18, have provided a piecewise solution for the MM reaction in terms of a fast transient solution for $s_{1}$, valid for $t \leq t_{c_{1}}$, as well as a QSS solution for $s_{1}$, valid for $t>t_{c_{1}}$ :

$$
\begin{aligned}
& s_{1}=s_{1}^{0}, \quad t \leq t_{c_{1}} \\
& s_{1}=K_{M_{1}} W\left[\sigma_{1} \exp \left(\sigma_{1}-\eta_{1} \hat{t}\right)\right], \quad t>t_{c_{1}}
\end{aligned}
$$

From the earlier work of Segel [22], we have a fast solution, valid when $t \leq t_{c_{1}}$, for the complex $c_{1}$, as well as a QSS solution which is valid for $t>t_{c_{1}}$,

$$
\begin{aligned}
& c_{1}=\bar{c}_{1}\left[1-\exp \left(-t / t_{c_{1}}\right)\right], \quad t<t_{c_{1}}, \quad \bar{c}_{1}=\frac{e_{1}^{0}}{K_{M_{1}}+s_{1}^{0}} s_{1}^{0} \\
& c_{1}=\frac{e_{1}^{0} s_{1}}{K_{M_{1}}+s_{1}}, \quad t \geq t_{c_{1}} .
\end{aligned}
$$

Collectively, equations 17a and 18b constitute an asymptotic solution that serves as an accurate approximation to the full time course of (11), provided the appropriate qualifiers (i.e, the RSA and the QSSA) are obeyed.

In addition to the timescale $t_{c_{1}}$, which quantifies the length of the initial fast transient (build-up of $c_{1}$ ), the time it takes for the majority of the substrate $s_{1}$ to be depleted is given by $t_{s_{1}}$. Although there are several methods for estimating the significant timescales of chemical reactions [23, we employ the heuristic method proposed by Segel 22, and approximate the depletion time to be effectively the total depletion of $s_{1}$ (the total depletion is $s_{1}^{0}$ ) divided by the maximum rate of substrate of depletion after $t_{c_{1}}$ :

$$
t_{s_{1}}=\frac{\Delta s_{1}}{\max _{0 \leq t}\left|\dot{s}_{1}\right|}=\frac{K_{M_{1}}+s_{1}^{0}}{V_{1}} .
$$


Generally speaking, $t_{s_{1}}$ is a reasonable measure of how long it takes for the non-observable reaction to complete.

\subsection{Geometrical picture of the enzyme catalyzed reaction, and conditions for} the validity of asymptotic solutions of the rate equations

While the asymptotic solutions are useful in that they can be employed to make certain predictions about the behavior of the reaction, asymptotic theory fails to yield a visual or geometric understanding of the dynamical behavior of the coupled enzyme auxiliary reaction mechanism. To paint a complete picture of the mathematical structure behind the reaction mechanism (1)-(2), we turn to dynamical systems theory, and analyze this problem from phase-space. From this perspective, after the initial buildup of $c_{1}$, the phase-space trajectory of the non-observable reaction 11a - 11b hugs a slow manifold, ' $\mathcal{M}_{\varepsilon}$ ', and is asymptotic to $\mathcal{M}_{\varepsilon}$ in the approach to equilibrium. The time it takes for the trajectory to reach the slow manifold is approximately $t_{c_{1}}$, while the time it takes for the trajectory to equilibrium is approximated by $t_{s_{1}}$. The condition for the validity of the asymptotic solution resides in how well the $c_{1}$-nullcline approximates the slow manifold, $\mathcal{M}_{\varepsilon}$, and also how straight the phase-space trajectory is in its approach to the slow manifold during the initial fast transient. The former of these conditions is known as the QSSA, and the latter is of course the geometrical interpretation of the $R S A$. We note that, chemically speaking, if the trajectory is close the slow manifold $\mathcal{M}_{\varepsilon}$, then the complex $C_{1}$ is assumed to be in a QSS for which the difference and the rate of $C_{2}$ depletion is approximately equal to the rate $C_{2}$ formation. Mathematically, it was originally proposed that the QSSA was valid if $t_{c_{1}} \ll t_{s_{1}}$,

$$
\frac{1}{k_{1}\left(K_{M_{1}}+s_{1}^{0}\right)} \ll \frac{K_{M_{1}}+s_{1}^{0}}{V_{1}} .
$$

In other words, it was assumed that the $c_{1}$-nullcline should be considered a good approximation to the slow manifold $\mathcal{M}_{\varepsilon}$ if the timescale accounting for the build-up of $c_{1}$ was small compared to the timescale accounting for the duration of the reaction. 
As for the validity of the RSA, Segel 24] proposed that one could assume little change in $s_{1}$ (an almost straight phase-space trajectory towards the slow manifold) if the depletion of $s_{1}$ over the timescale $t_{c_{1}}$ is minimal:

$$
\max _{t \geq 0}\left|\dot{s}_{1}\right| \cdot t_{c_{1}} \ll s_{1}^{0} .
$$

Since $\left|\dot{s}_{1}\right| \leq s_{1}^{0} e_{1}^{0}$, the strict inequality given in 21) translates to,

$$
\frac{e_{1}^{0}}{K_{M_{1}}+s_{1}^{0}} \equiv \varepsilon \ll 1 .
$$

Through scaling analysis, Segel 22 went on to show that the RSA, $\varepsilon \ll 1$, determines single-handedly the validity of the asymptotic solutions (17) and (18). Introducing the dimensionless variables $\hat{s}_{1}=s_{1} / s_{1}^{0}$ and $\hat{c}_{1}=c_{1} / \bar{c}_{1}$, Segel and Slemrod 24] demonstrated that, with respect to the dimensionless timescale $\tau=t / t_{c_{1}}$, equations 11a)-11a) scale as

$$
\begin{aligned}
& \frac{d \hat{s}_{1}}{d \tau}=\varepsilon\left[-\hat{s}_{1}+\frac{\sigma_{1}}{\sigma_{1}+1} \hat{c}_{1} \hat{s}_{1}+\frac{\kappa_{1}\left(1+\kappa_{1}\right)^{-1}}{\sigma_{1}+1} \hat{c}_{1}\right] \\
& \frac{d \hat{c}_{1}}{d \tau}=\hat{s}_{1}-\frac{\sigma_{1}}{\sigma_{1}+1} \hat{c}_{1} \hat{s}_{1}-\frac{1}{\sigma_{1}+1} \hat{c}_{1},
\end{aligned}
$$

where $\kappa_{1}=k_{-1} / k_{2}$ and $\varepsilon=e_{1}^{0} /\left(k_{M_{1}}+s_{1}^{0}\right)$. In contrast, under the timescale $T=t / t_{s_{1}}, 11 \mathrm{a}-11 \mathrm{a}$ become:

$$
\begin{aligned}
& \frac{d \hat{s}_{1}}{d T}=\left(\kappa_{1}+1\right)\left(\sigma_{1}+1\right)\left[-\hat{s}_{1}+\frac{\sigma_{1}}{\sigma_{1}+1} \hat{c}_{1} \hat{s}_{1}+\frac{\kappa_{1}\left(1+\kappa_{1}\right)^{-1}}{\sigma_{1}+1} \hat{c}_{1}\right] \\
& \varepsilon \frac{d \hat{c}_{1}}{d T}=\left(\kappa_{1}+1\right)\left(\sigma_{1}+1\right)\left[\hat{s}_{1}-\frac{\sigma_{1}}{\sigma_{1}+1} \hat{c}_{1} \hat{s}_{1}-\frac{1}{\sigma_{1}+1} \hat{c}_{1}\right] .
\end{aligned}
$$

Thus, it is apparent from the dimensionless equations $(23)-(24 \mathrm{~b}]$, that if $\varepsilon \ll 1$, then not only will the RSA hold, but the QSSA (which assumes that the $c_{1}$ nullcline is a good approximation to $\mathcal{M}_{\varepsilon}$ ) also holds. In fact, the RSA, $\varepsilon \ll 1$, is more restrictive than separation of timescales. After some algebraic calculations, 175 the separation of timescales, $t_{c_{1}} / t_{s_{1}} \ll 1$, can be written as:

$$
\frac{e_{1}^{0}}{K_{M_{1}}+s_{1}^{0}} \ll\left(1+\frac{K_{S_{1}}}{K_{1}}\right)\left(1+\frac{s_{1}^{0}}{K_{M_{1}}}\right),
$$


where $K_{S_{1}}=k_{-1} / k_{1}$, and $K_{1}=k_{2} / k_{1}$. For the RSA to be valid, the condition

$$
\frac{e_{1}^{0}}{K_{M_{1}}} \ll\left(1+\frac{s_{1}^{0}}{K_{M_{1}}}\right)
$$

must be satisfied, which is more stringent than condition (25), and hence dictates the conditions under which equation $(14 \mathrm{~b})$ or 16 can be applied. For this reason, it is nowadays considered that MM expressions are valid under the RSA (see Figures 1a and 1b), rather than the QSSA [25].

\subsection{Scaling analysis of the indicator reaction}

The scaling analysis of the indicator reaction requires knowledge of fast and slow timescales, as well as knowledge of reasonable upper and lower bounds of $s_{2}$ and $c_{2}$. We will start by trying to estimate a slow timescale for the indicator reaction. An accurate slow timescale should give us a reasonable estimation of the completion time for the indicator reaction (in the case of the auxiliary reaction, the completion of the indicator reaction can be faster, as fast, or slower than the non-observable reaction). For the non-observable reaction, the slow timescale is expressed in terms of the initial quantities $s_{1}^{0}$ and $e_{1}^{0}$, and the Michaelis constant $K_{M_{1}}$ :

$$
t_{s_{1}}=\frac{K_{M_{1}}+s_{1}^{0}}{V_{1}} .
$$

The quantity $e_{1}^{0}$ is the total amount of enzyme for the non-observable reaction. The construction of a homologous slow timescale for the indicator reaction is problematic in that the total amount of available enzyme $e_{2}^{A}$,

$$
e_{2}^{A}(t)=s_{1}^{0}-s_{1}-c_{1},
$$

is a time-dependent quantity. We will employ a mean-field approach to derive a slow (depletion) timescale for the indicator reaction. Let us first assume that we know the slow timescale for indicator reaction, and denote this timescale as $T_{s_{2}}$. Then, the mean available enzyme over the time course of the indicator reaction, which we will denote as $\left\langle e_{2}^{A}\right\rangle$, is given by

$$
\left\langle e_{2}^{A}\right\rangle=\frac{1}{T_{s_{2}}} \int_{0}^{T_{s_{2}}} e_{2}^{A}(t) \mathrm{d} t
$$




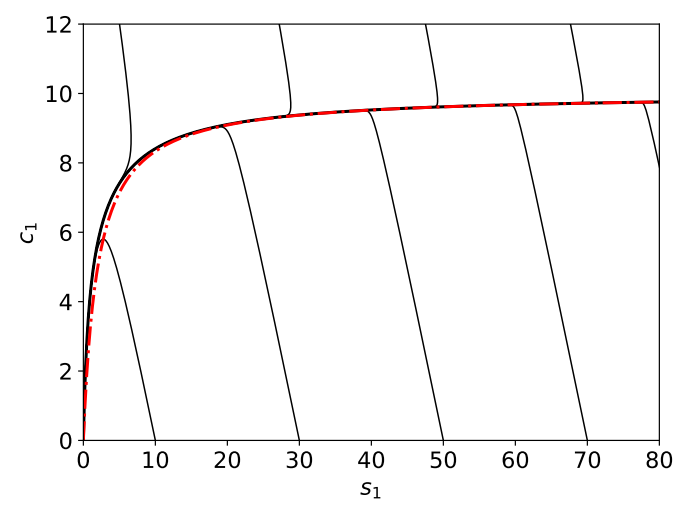

(a)



(b)

Figure 1: Geometrical picture of the single-substrate, single-enzyme non observable reaction (11). (a) Phase space dynamics with $e_{1}^{0}=10, k_{1}=1, k_{2}=1$ and $k_{-1}=1$. (b) Phase space dynamics with $e_{1}^{0}=1, s_{1}^{0}=78, k_{1}=1, k_{2}=5$ and $k_{-1}=1$. As $\varepsilon \rightarrow 0$, the accumulation of $c_{1}$ is more rapid, and the $c_{1}$-nullcline (dashed red curve) becomes a better approximation to the slow manifold, $\mathcal{M}_{\varepsilon}$, which is the thick black curve. The slow manifold curve is a graphical representation of the steady-state kinetic rate equation. The thin black curves are trajectories starting from different initial conditions, and represent the fast-transient kinetics of the reaction.

If the completion of the indicator reaction occurs long before the completion of the non-observable reaction, then we should expect that $\left\langle e_{2}^{A}\right\rangle \ll s_{1}^{0}$. In contrast, if the completion of the indicator reaction occurs long after the completion of the non-observable reaction, then would should expect $\left\langle e_{2}^{A}\right\rangle \approx s_{1}^{0}$. In any case, 
we can define the slow timescale as

$$
T_{s_{2}}=\frac{K_{M_{2}}+s_{2}^{0}}{k_{4}\left\langle e_{2}^{A}\right\rangle}
$$

which should yield a reasonable estimate for the slow timescale if the depletion of $s_{2}$ is influenced by a slow manifold.

Next, we want to scale the mass action equations that model the indicator reaction with respect to the quantities $\mathbb{T}=t / \hat{t}, s_{2}^{0}$, and $\max e_{2}^{A}$, where $\max e_{2}^{A}$ is the maximum amount of $e_{2}^{A}$ over the course of the indicator reaction:

$$
\max e_{2}^{A} \equiv \max _{t \leq T_{s_{2}}}\left(s_{1}^{0}-s_{1}-c_{1}\right)
$$

Utilizing $\max e_{2}^{A}$ as an upper bound on the available enzyme dictates a natural ${ }_{210}$ scaling of $c_{2}$,

$$
c_{2} \leq \frac{\max e_{2}^{A}}{K_{M_{2}}+s_{2}^{0}} s_{2}^{0} \equiv \hat{c}_{2} .
$$

Next, we scale the mass action equations with respect to the following dimensionless variables,

$$
\bar{s}_{2} s_{2}^{0}=s_{2}, \quad \bar{c}_{2} \hat{c}_{2}=c_{2}, \quad \bar{e}_{2}^{A} \max e_{2}^{A}=e_{2}^{A}, \quad \mathbb{T} \hat{t}=t,
$$

where $\hat{t}$ denotes an arbitrary timescale. Substitution of these quantities into the mass action equation yields

$$
\begin{aligned}
\frac{d \bar{s}_{2}}{d \mathbb{T}} & =\frac{\max e_{2}^{A}}{\left\langle e_{2}^{A}\right\rangle} \frac{\hat{t}}{T_{s_{2}}}\left(1+\kappa_{2}\right)\left(1+\sigma_{2}\right)\left[\left(\frac{\sigma_{2}}{1+\sigma_{2}} \bar{c}_{2}-\bar{e}_{2}^{A}\right) \bar{s}_{2}+\frac{\alpha}{1+\sigma_{2}} \bar{c}_{2}\right] \\
\lambda \frac{d \bar{c}_{2}}{d \mathbb{T}} & =\frac{\max e_{2}^{A}}{\left\langle e_{2}^{A}\right\rangle} \frac{\hat{t}}{T_{s_{2}}}\left(1+\kappa_{2}\right)\left(1+\sigma_{2}\right)\left[\left(\bar{e}_{2}^{A}-\frac{\sigma_{2}}{1+\sigma_{2}} \bar{c}_{2}\right) \bar{s}_{2}-\frac{1}{1+\sigma_{2}} \bar{c}_{2}\right] .
\end{aligned}
$$

In the above expressions, the dimensionless quantities $\sigma_{2}, \kappa_{2}$ and $\alpha$ are:

$$
\sigma_{2}=s_{2}^{0} / K_{M_{2}}, \quad \kappa_{2}=k_{-3} / k_{4}, \quad \alpha=\kappa_{2} /\left(1+\kappa_{2}\right) .
$$

The parameter $\lambda$, defined as

$$
\lambda=\frac{\max e_{2}^{A}}{K_{M_{2}}+s_{2}^{0}},
$$


is unique in that if it is sufficiently small, then it mathematically characterizes the indicator reaction as a singularly perturbed differential equation for which model reduction is possible through means of projecting onto the slow manifold, "M‥"

\section{Asymptotic analysis of the coupled auxiliary enzyme system}

Now that we have a good idea as to how the mass action equations of the indicator reaction scale, we want to try and find closed-form asymptotic solutions to the mass action equations or, at the very least, try and reduce the dimension of the mass action differential equations. The exact form of the scaled mass action equations will depend on the slow timescales of both the observable and non-observable indicator reactions. Thus, given that the respective slow timescale of the indicator and non-observable reactions are $T_{s_{2}}$ and $t_{s_{1}}$, we will analyze

$$
\begin{array}{r}
\frac{d \bar{s}_{2}}{d T}=\frac{\max e_{2}^{A}}{\left\langle e_{2}^{A}\right\rangle} \frac{\left(1+\kappa_{2}\right)\left(1+\sigma_{2}\right)}{\delta_{S}}\left[\left(\frac{\sigma_{2}}{\sigma_{2}+1} \bar{c}_{2}-\bar{e}_{2}^{A}\right) \bar{s}_{2}+\frac{\alpha}{\sigma_{2}+1} \bar{c}_{2}\right] \\
\lambda \frac{d \bar{c}_{2}}{d T}=\frac{\max e_{2}^{A}}{\left\langle e_{2}^{A}\right\rangle} \frac{\left(1+\kappa_{2}\right)\left(1+\sigma_{2}\right)}{\delta_{S}}\left[\left(\bar{e}_{2}^{A}-\frac{\sigma_{2}}{\sigma_{2}+1} \bar{c}_{2}\right) \bar{s}_{2}-\frac{1}{\sigma_{2}+1} \bar{c}_{2}\right],
\end{array}
$$

where $\delta_{S}$ is the ratio of the substrate depletion timescales, $\delta_{S}=T_{s_{2}} / t_{s_{1}}$, and $T=t / t_{s_{1}}$. Based on the scaling given in $37 \mathrm{a}$ and $37 \mathrm{~b}$ we will derive an estimate for $T_{s_{2}}$ as well as solutions for three particular cases, which are defined by the scale of $\delta_{S}$ : (i) Case 1: the indicator reaction is faster than the nonobservable reaction $\left(\delta_{S} \ll 1\right)$, Case 2 : the indicator reaction is roughly the same speed as the non-observable reaction $\left(\delta_{S} \approx 1\right)$, and Case 3: the indicator reaction is much slower than the non-observable reaction $\left(\delta_{S} \gg 1\right)$.

4.1. Case 1: The indicator reaction is faster than the non-observable reaction $\left(\delta_{S} \ll 1\right)$

If the indicator reaction is fast, and $\delta_{S} \ll 1$, then the dominant slow timescale is $t_{s_{1}}$, and thus the completion of the non-observable reaction will 
occur long after the completion of the indicator reaction. To start the analysis, we will rescale the mass action equations that govern the non-observable reaction with respect to $\hat{T}=t / T_{s_{2}}$ :

$$
\begin{aligned}
& \frac{d \hat{s}_{1}}{d \hat{T}}=\delta_{S}\left(1+\kappa_{1}\right)\left(1+\sigma_{1}\right)\left[-\hat{s}_{1}+\frac{\sigma_{1}}{\sigma_{1}+1} \hat{c}_{1} \hat{s}_{1}+\frac{\kappa_{1}\left(1+\kappa_{1}\right)^{-1}}{\sigma_{1}+1} \hat{c}_{1}\right] \\
& \varepsilon \frac{d \hat{c}_{1}}{d \hat{T}}=\delta_{S}\left(1+\kappa_{1}\right)\left(1+\sigma_{1}\right)\left[\hat{s}_{1}-\frac{\sigma_{1}}{\sigma_{1}+1} \hat{c}_{1} \hat{s}_{1}-\frac{1}{\sigma_{1}+1} \hat{c}_{1}\right] .
\end{aligned}
$$

By inspection of $38 \mathrm{a}$, if $\delta_{S} \ll 1$, then $s_{1}$ will be a slow variable over the $T_{s_{2}}$ timescale, and thus we will expect $s_{1}$ to be essentially constant over the time course of the indicator reaction. In addition, let us assume that $T_{s_{2}} \gg t_{c_{1}}$, in which case $c_{1}$ will be on the order of its maximum value on the $T_{s_{2}}$ timescale. Combining these observations leads to the approximation

$$
\begin{aligned}
& s_{1}=s_{1}^{0}+\mathcal{O}\left(\delta_{S}\right), \quad t \leq T_{s_{2}} \\
& c_{1}=\varepsilon s_{1}^{0}+\mathcal{O}\left(\delta_{S}\right), \quad t \leq T_{s_{2}}
\end{aligned}
$$

to the non-observable reaction over the timescale $T_{s_{2}}$. Equations 39a and (39b) seem to suggest that $e_{2}^{A} \ll 1$ over the $T_{s_{2}}$ timescale. Furthermore, since the changes in $s_{1}$ and $c_{1}$ are comparatively minimal when $t_{c_{1}} \leq t \leq T_{s_{2}}$, the production of $e_{2}^{A}$ is effectively constant over the $T_{s_{2}}$ timescale

$$
\dot{e}_{2}^{A} \approx \varepsilon k_{2} s_{1}^{0} \equiv \varpi
$$

Integration of 40 yields the following approximation of $e_{2}^{A}$ on the $T_{s_{2}}$ timescale

$$
e_{2}^{A} \approx \int_{0}^{t} \varpi \mathrm{d} u=\varpi t,
$$

where $u$ is a dummy variable. The approximate average value $\left\langle e_{2}^{A}\right\rangle$ on $T_{s_{2}}$ is easily obtainable through straightforward integration

$$
\left\langle e_{2}^{A}\right\rangle=\frac{\varpi}{T_{s_{2}}} \int_{0}^{T_{s_{2}}} t \mathrm{~d} t=\frac{1}{2} T_{s_{2}} \varpi
$$


and inserting 42 into 30 yields the following estimate for $T_{s_{2}}$ :

$$
T_{s_{2}}=\sqrt{\frac{2\left(K_{M_{2}}+s_{2}^{0}\right)}{k_{4} \varpi}} \equiv T_{s_{2}}^{*} .
$$

We can write 43 in a slightly more convenient form. Defining the limiting slow timescale $t_{s_{2}}^{*}$ as

$$
t_{s_{2}}^{*} \equiv \frac{K_{M_{2}}+s_{2}^{0}}{V_{2}}
$$

allows us to express $T_{s_{2}}^{\star}$ as

$$
T_{s_{2}}^{*}=\sqrt{2 t_{s_{1}} t_{s_{2}}^{*}}
$$

We expect $T_{s_{2}}^{*}$ to provide an accurate estimate for total completion time of the indicator reaction as long as the non-observable reaction is comparatively slow. For a generic (and linear) dynamical system of the form

$$
\dot{x}=-a x, \quad x(0)=x_{0}
$$

the depletion or characteristic timescale is $1 / a$, and thus we look for a timescale that is indicative of the time it takes for the initial quantity (i.e., $x_{0}$ in the context of (46) to deplete to an amount that is less than or equal to $x_{0} / e$. Following suit from the linear theory, we will consider the timescale $T_{s_{2}}^{*}$ to be a sufficient depletion timescale as long as

$$
s_{2}\left(T_{s_{2}}^{*}\right) \leq s_{2}^{0} / e \approx 0.37 s_{2}^{0}
$$

Numerical solutions of the mass action equations confirm the validity of the timescale $T_{s_{2}}^{*}$ when the indicator reaction is much faster than the non-observable reaction provided $t_{c_{1}} \ll T_{s_{2}}^{*}$ (see Figures $2 \mathrm{a}$ and $2 \mathrm{~b}$ ).

Next, we want to develop an asymptotic solution to the mass action equations that will be valid when: (1) $T_{s_{2}}^{*}$ is an accurate and precise depletion timescale, (2) the concentrations $s_{1}$ and $c_{1}$ remain on the order of their maximum values ( $s_{1}^{0}$ and $\varepsilon s_{1}^{0}$ respectively) for the duration of the indicator reaction, and (3) the fast timescale $t_{c_{1}}$ is negligibly short. To begin, let us assume that 


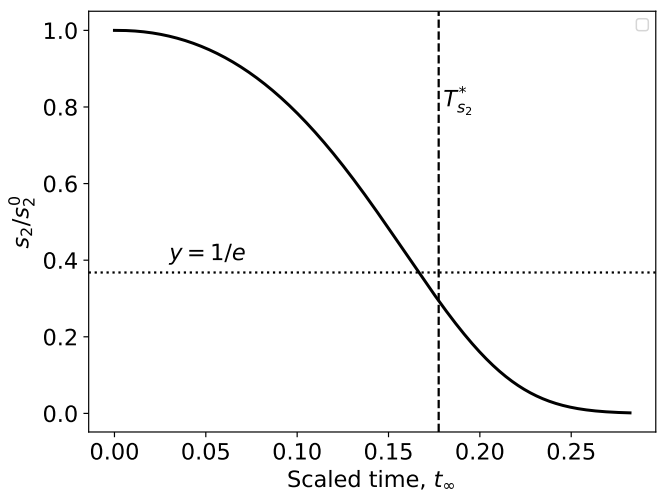

(a)

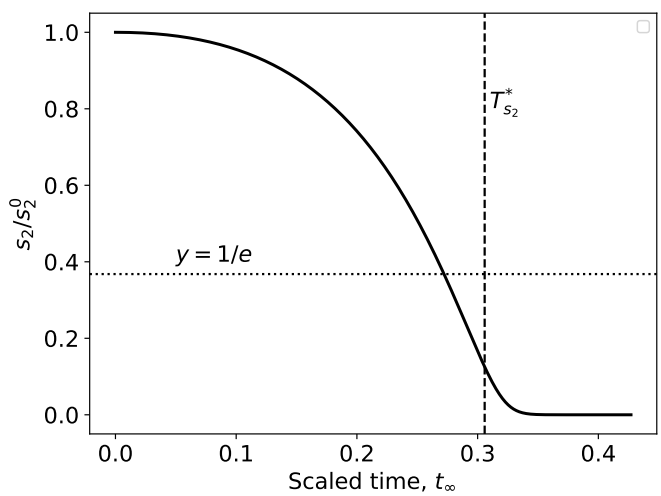

(b)

Figure 2: The accuracy of the timescale $T_{s_{2}}^{*}$ when the indicator reaction 2 is fast $\left(\delta_{S} \ll 1\right)$. The solid black curves numerical solutions to the mass action equations of the complete reaction. The dashed line marks the timescale $T_{s_{2}}^{*}$ and the dotted line represents the quantity $1 / e$. (a) The constants (without units) used in the numerical simulation are: $e_{1}^{0}=1, s_{1}^{0}=$ $100, k_{1}=1, k_{2}=1$ and $k_{-1}=1 . s_{2}^{0}=10, k_{3}=10, k_{4}=100$ and $k_{-3}=10$. (b) The constants (without units) used in the numerical simulation are: $e_{1}^{0}=1, s_{1}^{0}=100, k_{1}=1, k_{2}=1$ and $k_{-1}=1 . s_{2}^{0}=100, k_{3}=10, k_{4}=100$ and $k_{-3}=10$. In both cases, we see that the timescale $T_{s_{2}}^{*}$ yields an accurate approximation to the completion time of the indicator reaction. Time has been mapped to the $t_{\infty}$ scale: $t_{\infty}(t)=1-1 / \ln (t+e)$.

the initial concentration $s_{2}^{0}$ is large enough so that

$$
\max _{t \leq T_{s_{2}}^{*}} e_{2}^{A} \ll s_{2}^{0}
$$


in which case we can assume $\lambda \ll 1$. Then, from Tikhonov's theorem, and due to the existence of the slow manifold $\mathcal{M}_{\lambda}$, we have

$$
c_{2}=\frac{e_{2}^{A}}{K_{M_{2}}+s_{2}} s_{2}+\mathcal{O}(1)
$$

as a leading order approximation. Insertion of this approximation into the mass action equation for $s_{2}$ yields

$$
\dot{s}_{2}=-\frac{k_{4} e_{2}^{A}}{K_{M_{2}}+s_{2}} s_{2}+\mathcal{O}(1) .
$$

Substitution of $e_{2}^{A} \approx \varpi t$ into 50 gives us

$$
\dot{s}_{2}=-\frac{k_{4} \varpi t}{K_{M_{2}}+s_{2}} s_{2}+\mathcal{O}(1)
$$

as our final asymptotic approximation to the differential equations governing the temporal depletion of $s_{2}$. Equation (51) has a closed-form solution in the form of the Schnell-Mendoza equation

$$
s_{2}=K_{M_{2}} W\left[\sigma_{2} \exp \left(\sigma_{2}-\frac{k_{4} \varpi t^{2}}{2 K_{M_{2}}}\right)\right]
$$

and provides an accurate approximation to the mass action model (see FIGUREs $3 \mathrm{a}$ and $3 \mathrm{~b}$.

4.2. Case 2: The indicator reaction is roughly the same speed as the nonobservable reaction $(\delta \approx 1)$

It is instinctive, in the case that the non-observable reaction and the indicator reaction both complete at roughly the same time, to use either the slow timescale, $t_{s_{1}}$ or $T_{s_{2}}$, as the depletion timescale for the complete reaction. Of course, given our earlier definition of the timescale $T_{s_{2}}$

$$
T_{s_{2}}=\frac{K_{M_{2}}+s_{2}^{0}}{k_{4}\left\langle e_{2}^{A}\right\rangle},
$$

we can formulate a nonlinear algebraic equation that will allow us to compute 275 an estimate for the depletion timescale when the reactions are equivalent in 


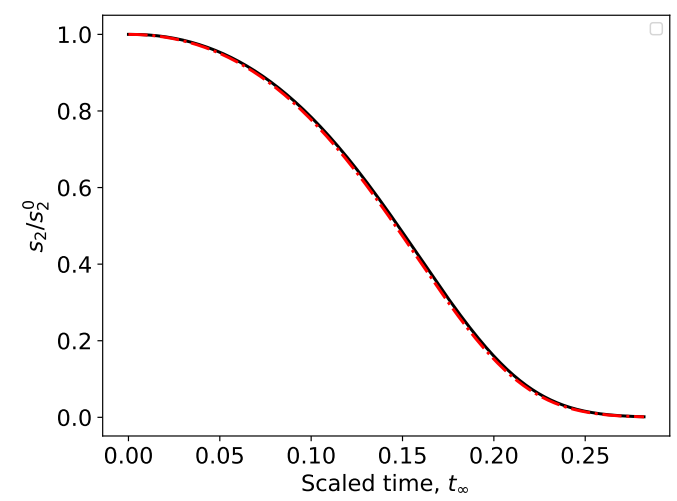

(a)



(b)

Figure 3: The leading order asymptotic solution 52 of the substrate concentration for the indicator reaction matches the numerical solution when the indicator reaction is faster than the non-observable reaction $\left(\delta_{S} \ll 1\right)$. The solid black curves numerical solutions to the mass action equations of the complete reaction 8 and the broken red curves are numerical solutions to the asymptotic differential equation [51). (a) The constants (without units) used in the numerical simulation are: $e_{1}^{0}=1, s_{1}^{0}=100, k_{1}=1, k_{2}=1$ and $k_{-1}=1 . s_{2}^{0}=10$, $k_{3}=10, k_{4}=100$ and $k_{-3}=10$. (b) The constants (without units) used in the numerical simulation are: $e_{1}^{0}=1, s_{1}^{0}=100, k_{1}=1, k_{2}=1$ and $k_{-1}=1 . s_{2}^{0}=100, k_{3}=10, k_{4}=100$ and $k_{-3}=10$. Time has been mapped to the $t_{\infty}$ scale: $t_{\infty}(t)=1-1 / \ln (t+e)$.

speed. First,

$$
\left\langle e_{2}^{A}\right\rangle=\frac{1}{T_{s_{2}}} \int_{0}^{T_{s_{2}}}\left(s_{1}^{0}-s_{1}-c_{1}\right) \mathrm{d} t
$$


and thus we see that $T_{s_{2}}$ should satisfy

$$
\int_{0}^{T_{s_{2}}}\left(s_{1}^{0}-s_{1}-c_{1}\right) \mathrm{d} t=\frac{K_{M_{2}}+s_{2}^{0}}{k_{4}} .
$$

Second, under the RSA, the concentration $c_{1}$ is expressible (algebraically) in terms of $s_{1}$, and therefore

$$
\int_{0}^{T_{s_{2}}}\left(s_{1}^{0}-s_{1}-c_{1}\right) \mathrm{d} t \approx \int_{0}^{T_{s_{2}}} \frac{\left(K_{M_{1}}+s_{1}\right) \Delta s_{1}-e_{1}^{0} s_{1}}{K_{M_{1}}+s_{1}} \mathrm{~d} t
$$

280

where $\Delta s_{1}=s_{1}^{0}-s_{1}$ (the timescale $t_{c_{1}}$ has been assumed to be negligibly small and hence left out of the integrand, although it is straightforward to include this term). Third, the definite integral on the right hand side of $\sqrt[56]{ }$ is straightforward to compute analytically; evaluating it will yield a nonlinear equation in terms of the variable $T_{s_{2}}$, and the solution to 55 can be approximated by using a standard contraction mapping algorithm. Using the average $\left\langle e_{2}^{A}\right\rangle$ provides an accurate estimate of the slow (depletion) timescale (see Figure 4 .

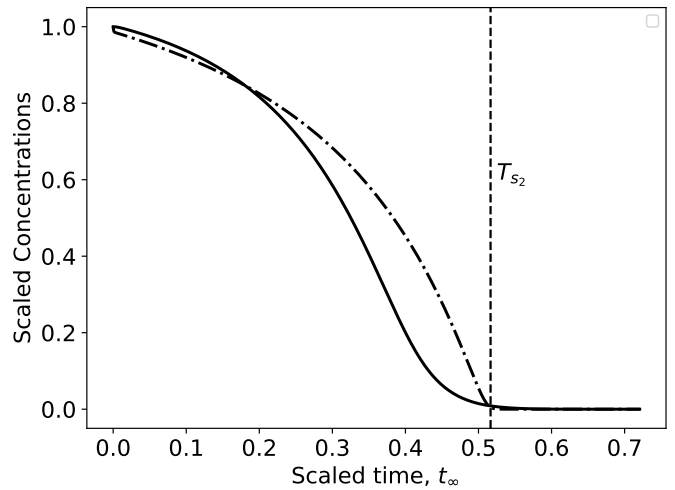

Figure 4: The averaging method for the estimation of the depletion timescale for the indicator substrate is still valid when the non-observable and indicator reactions occur at roughly the same speed $\left(\delta_{S} \approx 1\right)$. The solid black curve is the numerically-computed depletion curve of $s_{2}$ and the dotted/dashed black curve is the numerically-integrated depletion curve of $s_{1}$. In this numerical simulation $k_{3}=1, k_{4}=1, k_{-3}=10, s_{2}^{0}=70$, and $k_{1}=10, k_{2}=15, k_{-1}=1, e_{1}^{0}=1$ and $s_{1}^{0}=70$. Both substrates have been scaled as $s_{2} / s_{2}^{0}$ and $s_{1} / s_{1}^{0}$. Time has been mapped to the $t_{\infty}$ scale: $t_{\infty}(t)=1-1 / \ln (t+e)$. 
From a practical point of view, the utility in estimating $T_{s_{2}}$ through a contraction mapping is rather minimal. The objective here will be to construct a criteria from which a reduced model can be be extracted from the mass action equations that will be valid without any a priori knowledge of the intrinsic timescales of the indicator reaction (or the non-observable reaction). To do this, let us first revisit the generic scaling introduced in the previous section:

$$
\begin{aligned}
\frac{d \bar{s}_{2}}{d T} & =\frac{\max e_{2}^{A}}{\left\langle e_{2}^{A}\right\rangle} \frac{\left(1+\kappa_{2}\right)\left(1+\sigma_{2}\right)}{\delta_{S}}\left[\left(\frac{\sigma_{2}}{1+\sigma_{2}} \bar{c}_{2}-\bar{e}_{2}^{A}\right) \bar{s}_{2}+\frac{\alpha}{1+\sigma_{2}} \bar{c}_{2}\right] \\
\lambda \frac{d \bar{c}_{2}}{d T} & =\frac{\max e_{2}^{A}}{\left\langle e_{2}^{A}\right\rangle} \frac{\left(1+\kappa_{2}\right)\left(1+\sigma_{2}\right)}{\delta_{S}}\left[\left(\bar{e}_{2}^{A}-\frac{\sigma_{2}}{1+\sigma_{2}} \bar{c}_{2}\right) \bar{s}_{2}-\frac{1}{1+\sigma_{2}} \bar{c}_{2}\right] .
\end{aligned}
$$

Bearing in mind that it is assumed that $\delta_{S} \approx 1$, it is sufficient (but not necessary) to bound $\lambda$ in order to assemble a dynamical model that can be reduced (asymptotically) through slow manifold projection. The upper bound on $\lambda$, which we denote as $\lambda^{\max }$, is

$$
\lambda \leq \lambda^{\max } \equiv \frac{s_{1}^{0}}{K_{M_{2}}+s_{2}^{0}} .
$$

The parameter $\lambda^{\max }$ is the natural small parameter when the indicator is very slow. Furthermore, if the non-observable reaction completes very quickly relative to the non-observable reaction, and $\delta_{S} \ll 1$, then the average available enzyme should be on the order of $s_{1}^{0}$ :

$$
\left\langle e_{2}^{A}\right\rangle=\frac{1}{T_{s_{2}}} \int_{0}^{T_{s_{2}}} e_{2}^{A} \mathrm{~d} t \sim s_{1}^{0} .
$$

Thus, if $s_{2}^{0} \gg s_{1}^{0}$, then the approximation

$$
\dot{s}_{2}=-\frac{k_{4} e_{2}^{A}}{K_{M_{2}}+s_{2}} s_{2}+\mathcal{O}(1)
$$

will be valid regardless of the relative speeds of the reactions when $\lambda^{\max } \ll 1$. Furthermore, 60 admits a closed-form solution using separation of variables that consists of composite Lambert- $W$ functions (we do not present this expression here, although we remark that it is straightforward, albeit somewhat 
tedious to derive). Under the RSA, we obtain

$$
\dot{s}_{2}=-\left(\frac{\left(K_{M_{1}}+s_{1}\right) \Delta s_{1}-e_{1}^{0} s_{1}}{K_{M_{1}}+s_{1}}\right)\left(\frac{k_{4}}{K_{M_{2}}+s_{2}}\right) s_{2}
$$

as the final form of our reduced differential equation for $\dot{s}_{2}$.

\subsection{Case 3: The indicator reaction is much slower than the non-observable} reaction $\left(\delta_{S} \gg 1\right)$

We now consider the case when $\delta_{S} \gg 1$, and the completion of the nonobservable occurs much sooner than the completion of the indicator reaction. As mentioned in the previous subsection, a very slow indicator reaction suggests that $s_{2}$ will be slow over the timescale $t_{s_{1}}$. Consequently, we can approximate $s_{2}$ as

$$
s_{2}=s_{2}^{0}, \quad t<t_{s_{1}} \text {. }
$$

Furthermore, because the non-observable reaction has effectively completed when $t=t_{s_{1}}$, we can approximate $\Delta s_{1}=s_{1}^{0}$ when $t \geq t_{s_{1}}$, in which case

$$
\dot{s}_{2}=-\frac{k_{4} s_{1}^{0}}{K_{M_{2}}+s_{2}} s_{2}+\mathcal{O}(1), \quad t \geq t_{s_{1}} .
$$

Equation (63) can be integrated directly to yield a Schnell-Mendoza equation for $s_{2}$ :

$$
s_{2}=K_{M_{2}} W\left[\sigma_{2} \exp \left(\sigma_{2}-\eta_{2}(t)\right)\right], \quad t \geq t_{s_{1}} .
$$

The validity of the approximate solution 62 can be established by the mathematical formulation of the RSA for the indicator reaction. If $s_{2} \approx s_{2}^{0}$ over the interval $\left[0, t_{s_{1}}\right]$, then

$$
\max _{t \leq t_{s_{1}}}\left|\dot{s}_{2}\right| \cdot t_{s_{1}} \ll s_{2}^{0}
$$

The inequality given in 65 translates to

$$
\delta_{S} \gg\left(\sigma_{2}+1\right)\left(\kappa_{2}+1\right),
$$

with $\max \dot{s}_{2}=k_{3} s_{1}^{0} s_{2}^{0}$. In the case of a slow indicator reaction, we expect that $T_{s_{2}}=t_{s_{2}}^{*}$. Thus, we have a RSA that is pertinent to the indicator reaction

$$
\frac{V_{1}}{V_{2}} \gg \frac{K_{M_{1}}}{K_{M_{2}}}\left(1+\sigma_{1}\right)\left(1+\kappa_{2}\right)
$$


320 during the initial build-up of $c_{2}$ when $t \leq t_{s_{1}}$. Equation 67 is analogous to the term used to measure the strength of fully competitive enzyme reactions with alternative substrates [26, 27]. Numerical simulations (see Figure 5] confirm the validity of $t_{s_{2}}^{*}$ and 63 .

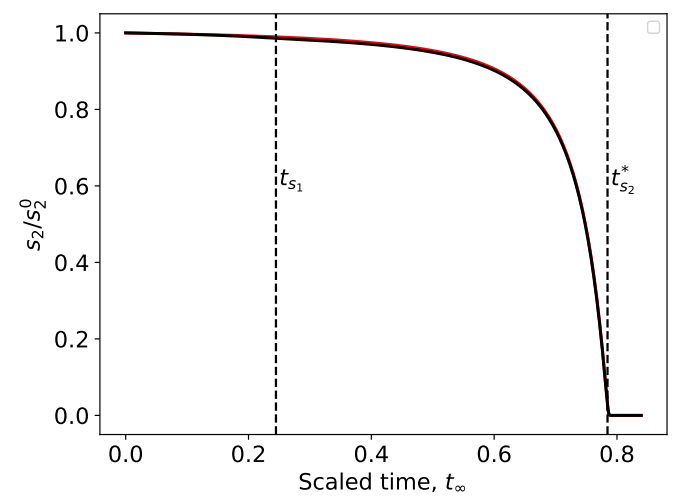

Figure 5: Validity of the timescale $t_{s_{2}}^{*}$ and the reduced ordinary differential equation given by 63 for the substrate depletion of the indicator reaction when the indicator reaction is much slower than the non-observable reaction $\left(\delta_{S} \gg 1\right)$. The solid black curve in the numerical solution to the mass action equations $(8)$ and the solid red curve corresponds to the numerical solution to 63 extended to $t \geq 0$. In this numerical simulation $k_{3}=0.1, k_{4}=1, k_{-3}=$ $10, s_{2}^{0}=10000$, and $k_{1}=25, k_{2}=100, k_{-1}=1, e_{1}^{0}=1$ and $s_{1}^{0}=100$. The respective values of $\lambda^{\max }$ and $\delta_{S}$ are $\approx 0.009$ and $\approx 0.01$. Time has been mapped to the $t_{\infty}$ scale: $t_{\infty}(t)=1-1 / \ln (t+e)$.

\section{Estimate of lag time of the indicator reaction}

Up until this point, we have not mentioned the equivalent of a fast timescale that is pertinent to the indicator reaction. In the case of the non-observable reaction, the fast timescale corresponds to the time it takes the reaction to reach QSS. However, based on our scaling analysis, we have demonstrated that a QSSA can be imposed for the complete duration of the reaction as long as $\lambda \ll 1$. At first glance, it would seem that the kinetics of the indicator reaction omit the influence of a fast timescale. This is however false. To derive the fast 
timescale, we will assume that initial conditions are not experimental, and that the indicator reaction is equipped with a non-trivial amount of complex $c_{2}^{0}$ at the start of the reaction. Furthermore, we will assume that the substrate, $s_{2}$ does not deplete significantly over the duration of the fast timescale. Denoting the fast timescale as $t_{c_{2}}$, the mass action equation can be linearized:

$$
\begin{aligned}
\dot{c}_{2} & =k_{3}\left(e_{2}^{A}-c_{2}\right) s_{2}^{0}-\left(k_{-3}+k_{4}\right) s_{2}^{0}, \quad t \leq t_{c_{2}} \\
c_{2}(0) & =c_{2}^{0} .
\end{aligned}
$$

The solution to the linear equation $(68)$ is given by Duhamel's Principle

$$
c_{2}(t)=\exp (-\mu t) c_{2}^{0}+\int_{0}^{t} \exp (\mu(s-t)) e_{2}^{A}(s) d s, \quad \mu \equiv k_{3}\left(K_{M_{2}}+s_{2}^{0}\right),
$$

and thus, the naturally occurring characteristic timescale is $1 / \mu$ :

$$
t_{c_{2}} \equiv \frac{1}{k_{3}\left(K_{M_{2}}+s_{2}^{0}\right)} .
$$

If initial conditions are not experimental, then $t_{c_{2}}$ provides a reasonable estimate on the time it takes for the indicator reaction to reach QSS. However, since typical experimental initial conditions start on the $c_{2}$-nullcline, we turn to scaling to provide a biochemical interpretation of the timescale $t_{c_{2}}$. Defining $T^{*}=t / t_{c_{2}}$, we obtain:

$$
\begin{aligned}
& \frac{d \bar{s}_{2}}{d T^{*}}=\lambda\left[\left(\frac{\sigma_{2}}{1+\sigma_{2}} \bar{c}_{2}-\bar{e}_{2}^{A}\right) \bar{s}_{2}+\frac{\alpha}{1+\sigma_{2}} \bar{c}_{2}\right] \\
& \frac{d \bar{c}_{2}}{d T^{*}}=\left(\bar{e}_{2}^{A}-\frac{\sigma_{2}}{1+\sigma_{2}} \bar{c}_{2}\right) \bar{s}_{2}-\frac{1}{1+\sigma_{2}} \bar{c}_{2} .
\end{aligned}
$$

We see from the scaling that $t_{c_{2}}$ defines a lag timescale when experimental initial conditions are prescribed. Over this timescale, the indicator reaction is effectively stationary, since both $c_{2}$ and $s_{2}$ are slow variables $\left(c_{2}\right.$ is a slow variable not from scaling, but because it will stay near the $c_{2}$-nullcline over short timescales, and thus $\dot{c}_{2} \approx 0$ when $t \leq t_{c_{2}}$ ). Thus, the fast timescale of the indicator reaction defines the lag time of the reaction. The lag time is present 
due to the fact that the indicator reaction cannot "start" until after a non-trivial amount of enzyme $E_{2}$ has been produced in the non-observable reaction.

The relationship between $\lambda, t_{c_{2}}$ and $T_{s_{2}}$ is now evident. The ratio of fast and slow timescales is bounded above by $\lambda$,

$$
\frac{t_{c_{2}}}{T_{s_{2}}}<\lambda .
$$

The strict inequality follows from the fact that,

$$
\frac{t_{c_{2}}}{T_{s_{2}}}=\frac{\bar{\lambda}}{\left(1+\sigma_{2}\right)\left(1+\kappa_{2}\right)}
$$

where $\bar{\lambda}$ is given by

$$
\bar{\lambda} \equiv \frac{\left\langle e_{2}^{A}\right\rangle}{K_{M_{2}}+s_{2}^{0}} .
$$

340 Furthermore, since $\left\langle e_{2}^{A}\right\rangle \leq \max e_{2}^{A}$, we have that

$$
\bar{\lambda} \leq \lambda
$$

from which 72 follows.

To explore the relationship between the QSSA and the RSA, we note that the parameter $\lambda^{\max }$ is easily derived using Segel's heuristic approach [22]:

$$
\max \left|\dot{s}_{2}\right| \cdot t_{c_{2}} \ll s_{2}^{0} \rightarrow \lambda^{\max } \ll 1
$$

Since it is clear that

$$
\bar{\lambda} \leq \lambda \leq \lambda^{\max }
$$

it follows that the RSA (i.e., $\lambda^{\max } \ll 1$ ) ensures separation of fast and slow timescales; consequently, the RSA for the indicator reaction implies the QSSA and is thus a universal qualifier for the validity of the reduced model.

\section{Discussion}

The primary contribution of this paper is to introduce methods for the appropriate scaling and timescale estimates of the auxiliary enzyme assay. The 
identification of specific parameters through scaling has yielded necessary and sufficient conditions for the QSSA, whereas previous nonlinear studies of the coagulation cascade with auxiliary enzymes have employed the QSSA without justification [10]. Moreover, previous analyses [8] dot not provide insight as to 355 how to properly estimate kinetic timescales via nonlinear methods, even though the coupled enzyme auxiliary assays are inherently nonlinear. This work outlines a clear procedure for estimating depletion timescales, and serves as a template for the analysis of more complicated reactions. We give a brief summary of the results of the analysis in what follows.

Scaling analysis of the mass action equations that model the kinetics of a coupled auxiliary enzyme reaction (1)-(2) has revealed two small parameters, $\varepsilon$ and $\lambda^{\max }$,

$$
\begin{aligned}
\lambda^{\max } & =\frac{s_{1}^{0}}{K_{M_{2}}+s_{2}^{0}} \ll 1 \\
\varepsilon & =\frac{e_{1}^{0}}{K_{M_{1}}+s_{1}^{0}} \ll 1 .
\end{aligned}
$$

The parameters $\varepsilon$ and $\lambda$ regulate the partition of the slow timescales $t_{c_{1}}, t_{c_{2}}$ and the fast timescales $t_{s_{1}}$ and $T_{s_{2}}$ :

$$
\frac{t_{c_{1}}}{t_{s_{1}}}<\varepsilon, \quad \frac{t_{c_{2}}}{T_{s_{2}}}<\lambda \leq \lambda^{\max }
$$

When these parameters are small, and the timescales $t_{c_{2}}$ and $t_{s_{1}}$ are adequately separated, the indicator reaction can be assumed to be in a QSS for the duration of the reaction (i.e, for $t \geq 0$ ). There is a twofold reasoning to this assumption. First, if $\lambda^{\max } \ll 1$, then

$$
\lambda \equiv \frac{\max e_{2}^{A}}{K_{M_{2}}+s_{2}^{0}} \ll 1,
$$

and model reduction from slow manifold projection is valid regardless of which reaction finishes first (non-observable or indicator). Since it is not generally possible to determine which reaction is faster in the typical experiment a priori, the condition that $\lambda^{\max } \ll 1$ serves as a sufficient qualifier to ensure the validity 
of the reduced model for the reaction rate of depletion of the indicator substrate:

$$
\dot{s}_{2}=-\left(\frac{\left(K_{M_{1}}+s_{1}\right) \Delta s_{1}-e_{1}^{0} s_{1}}{K_{M_{1}}+s_{1}}\right)\left(\frac{k_{4}}{K_{M_{2}}+s_{2}}\right) s_{2} .
$$

Second, as long as $t_{c_{2}} \ll t_{s_{1}}$, a QSSA will effectively hold for all time since experimental initial conditions lie on the $c_{2}$-nullcline. From the theory of singular perturbations, the slow manifold, " $\mathcal{M}_{\lambda}$," is an invariant manifold that is well-approximated by the $c_{2}$-nullcline when $\lambda^{\max } \ll 1$. Because experimental initial conditions lie on the $c_{2}$-nullcline, the phase-space trajectory is already extremely close to the slow manifold, and therefore there is no need for an initial fast transient in order for the trajectory to reach the slow manifold. As we have pointed out, the slow manifolds is a geometrical representation of the steadystate rate equation for the reaction. Note that this is very different from the non-observable reaction, since a fast transient (the duration of the fast transient is approximated by the timescale $t_{c_{1}}$ ) must elapse before the QSSA is justifiable.

In addition, simple asymptotic solutions to the mass action equations were derived that are valid when the indicator reaction is very fast or very slow in comparison to the non-observable reaction. If the indicator reaction is fast, then the time course of the indicator substrate $s_{2}$ is accurately model by

$$
s_{2}=K_{M_{2}} W\left[\sigma_{2} \exp \left(\sigma_{2}-\frac{k_{4} \varpi t^{2}}{2 K_{M_{2}}}\right)\right],
$$

where $W$ denotes the Lambert- $W$ function. In contrast, if the indicator reaction is very slow, then the time course of $s_{2}$ can be modeled by

$$
s_{2}=K_{M_{2}} W\left[\sigma_{2} \exp \left(\sigma_{2}-\frac{V_{2} t}{K_{M_{2}}}\right)\right] .
$$

Note that the above two expressions are analogous to the Schnell-Mendoza equation [18].

It should be pointed out that the condition $\lambda^{\max } \ll 1$, which can be ensured by requiring an excess of the initial amount of substrate $s_{2}$ (i.e., requiring that $s_{2}^{0}$ be large enough so that $s_{1}^{0} \ll s_{2}^{0}$ ), is sufficient but not necessary for the 
validity of the reduced model presented in (61). In general, it is desirable that $s_{2}^{0}$ be much larger than the maximum of amount of $e_{2}^{A}$ over the timescale of the indicator reaction. If the indicator reaction is fast, then the maximum amount of available enzyme, $\max e_{2}^{A}$, will be small, and thus the requirement that $s_{1}^{0} \ll s_{2}^{0}$ is unnecessary as if $\max e_{2}^{A} \ll K_{M_{2}}$ (see Figure 6 ). Of course, the integrity of the reduced model does not diminish if $s_{1}^{0} \ll s_{2}^{0}$; therefore, the qualifier, $\lambda^{\max } \ll 1$, is a universal condition for the validity of [61, as this will ensure that $\max e_{2}^{A} \ll s_{2}^{0}$ on any timescale.



Figure 6: The condition that $\lambda \ll 1$ is necessary for slow manifold projection, while the condition $\lambda^{\max } \ll 1$ is merely sufficient. The solid black curve is the numerical solution to the mass action equations and the broken red curve is to the numerical solution to 61). In this simulation $k_{3}=1, k_{4}=100, k_{-3}=10, s_{2}^{0}=1$, and $k_{1}=1, k_{2}=1, k_{-1}=1, e_{1}^{0}=1$ and $s_{1}^{0}=100 . s_{1}^{0} / s_{2}^{0} \approx 100$ and $\lambda^{\max } \approx 1$. However, $\max e_{2}^{A} \approx 1.543$ and therefore $\lambda \approx 0.014 \ll 1$. Time has been mapped to the $t_{\infty}$ scale: $t_{\infty}(t)=1-1 / \ln (t+e)$.

Finally, three reduced models have been derived that can be utilized in the inverse problem. Our analysis seems suggests that a fast indicator reaction is the most beneficial case for parameter estimation. Under this circumstance, two expressions

$$
s_{2}=K_{M_{2}} W\left[\sigma_{2} \exp \left(\sigma_{2}-\frac{V_{2} V_{1} t^{2}}{2 K_{M_{2}}\left(s_{1}^{0}+K_{M_{1}}\right)}\right)\right]
$$


and

$$
s_{2}=-\int_{t_{c_{1}}}^{t}\left(\frac{\left(K_{M_{1}}+s_{1}\right) \Delta s_{1}-e_{1}^{0} s_{1}}{K_{M_{1}}+s_{1}}\right)\left(\frac{k_{4}}{K_{M_{2}}+s_{2}} s_{2}\right) \mathrm{d} u
$$

can be simultaneously utilized to estimate the four unknown parameters $V_{1}, V_{2}, K_{M_{1}}$, and $K_{M_{2}}$. The full analysis of the inverse problem is beyond the scope of this paper; we hope, however, to theoretically investigate the scope of parameter estimation in coupled auxiliary enzyme assays in subsequent future work.

\section{Acknowledgements}

We are grateful to Dr. Enrico DiCera (Saint Louis University School of Medicine) for his suggestions to explore this problem. We are also grateful to Dr. Antonio Baici (University of Zurich) for helpful discussions about this work during the 2017 Beilstein Enzymology Symposia (Rüdesheim, Germany). This work is partially supported by the University of Michigan Protein Folding Diseases Initiative, and Beilstein-Institut zur Förderung der Chemischen Wissenschaften through its Beilstein Enzymology Symposia. Dr. Stroberg is a fellow of the Michigan IRACDA program (NIH/NIGMS grant: K12 GM111725).

\section{References}

[1] F. B. Rudolph, B. W. Baugher, R. S. Beissner, Techniques in coupled enzyme assays, Methods Enzymol. 63 (1979) 22-42.

[2] B. A. C. Storer, A. Cornish-bowden, P. O. Box, B. Birmingham, The kinetics of coupled enzyme reactions, Biochem. J. 141 (1974) 205-209.

[3] W. W. Cleland, Optimizing coupled enzyme assays, Anal. Biochem. 99 (1979) 142-145.

[4] W. R. McClure, Kinetic analysis of coupled enzyme assays, Biochemistry 8 (1969) 2782-2786.

[5] J. S. Easterby, Coupled enzyme assays: A general expression for the transient, Biochim Biophys Acta. 293 (1973) 552-558. 
[15] M. S. Okino, M. L. Mavrovouniotis, Simplification of mathematical models in chemical reaction systems, Chem. Rev. 98 (1998) 391-408.

[16] R. Bertram, J. E. Rubin, Multi-timescale systems and fast-slow analysis, Math. Biosci. 287 (2017) 105-121. 
[17] S. Schnell, C. Mendoza, The condition for pseudo-first-order kinetics in enzymatic reactions is independent of the initial enzyme concentration, Biophys. Chem. 107 (2004) 165-174.

[18] S. Schnell, C. Mendoza, Closed form solution for time-dependent enzyme kinetics, J. Theor. Biol. 187 (1997) 207-212.

[19] A. H. Nguyen, S. J. Fraser, Geometrical picture of reaction in enzyme kinetics, J. Chem. Phys. 91 (1989) 186-193.

[20] M. R. Roussel, S. J. Fraser, Geometry of the steady-state approximation: Perturbation and accelerated convergence methods, J. Chem. Phys. 93 (1990) 1072-1081.

[21] S. M. Hanson, S. Schnell, Reactant stationary approximation in enzyme kinetics, J. Phys. Chem. A 112 (2008) 8654-8658.

[22] L. A. Segel, On the validity of the steady state assumption of enzyme kinetics, Bull. Math. Biol. 50 (1988) 579-593.

[23] S. K. Shoffner, S. Schnell, Approaches for the estimation of timescales in nonlinear dynamical systems: Timescale separation in enzyme kinetics as a case study, Math. Biosci. 287 (2017) 122-129.

[24] L. A. Segel, M. Slemrod, The quasi-steady-state assumption: a case study in perturbation, SIAM Rev. 31 (1989) 446-477.

[25] S. Schnell, Validity of the Michaelis-Menten equation - Steady-state, or reactant stationary assumption: that is the question, FEBS J. 281 (2014) 464-472.

[26] S. Schnell, C. Mendoza, Time-dependent closed form solutions for fully competitive enzyme reactions, Bull. Math. Biol. 62 (2000) 321-336.

[27] S. Schnell, C. Mendoza, Enzyme kinetics of multiple alternative substrates, J. Math. Chem. 27 (2000) 155-170. 